\title{
THE SDFEM FOR A CONVECTION-DIFFUSION PROBLEM WITH TWO SMALL PARAMETERS
}

\author{
HANS-GÖRG ROOS \\ Technische Universität Dresden, Germany \\ ZORICA UZELAC ${ }^{1}$ \\ Faculty of Technical Sciences, Novi Sad, Yugoslavia \\ Dedicated to John J.H.Miller on the occasion of his 65th birthday.
}

\begin{abstract}
A singularly perturbed convection-diffusion problem with two small parameters is considered. The problem is solved using the streamline-diffusion finite element method on a Shishkin mesh. We prove that the method is convergent independently of the perturbation parameters. Numerical experiments support these theoretical results.
\end{abstract}

2000 Mathematics Subject Classification: 34E15, 65L10, 65L12.

Keywords: convection-diffusion problems, singular perturbation, streamline-diffusion method, two small parameters, Shishkin-type mesh.

\section{Introduction}

We consider the singularly perturbed convection-diffusion problem

$$
L u:=-\varepsilon_{1} u^{\prime \prime}+\varepsilon_{2} b(x) u^{\prime}+c(x) u=f(x) \quad x \in I=(0,1), \quad u(0)=0, \quad u(1)=0,
$$

where $b, c, f$ are smooth functions, $b(x) \geqslant b_{0}>0, c(x) \geqslant c_{0}>0$ and $0<\varepsilon_{1,2}<<1$ are both small perturbation parameters. Although physical problems frequently involve the solution of boundary-value problems with many small parameters, not much is known about robust numerical methods for solving two parameter problems ( see O'Malley [8] for some physical problems involving two small parameters and for details concerning asymptotic expansions).

Shishkin and Titov [12] proved, for an exponentially fitted difference scheme on an equidistant mesh, for some $\nu<2 / 5$, that

$$
\left|u\left(x_{i}\right)-u_{i}\right| \leqslant C h^{\nu}
$$

where $\mathrm{C}$ is independent of $\varepsilon$ and $\mathrm{h}$. Instead of using exponential fitting, the use of layeradapted meshes becomes more and more popular. Vulanovic [14] considers Bakhvalov and

${ }^{1}$ This paper was written during a visit to the Technical University of Dresden in September-October 2002 supported by DAAD grant and partly supported by Ministry of Science of the Republic of Serbia under grant no 1840 . 
Shishkin meshes but assumes $\varepsilon_{2}=\varepsilon_{1}^{\frac{1}{2}+p}$ with $p>0$. Then the problem (1.1) behaves similarly as the standard reaction-diffusion problem with $\varepsilon_{2}=0$. Two-parameter problems are considered also in $\mathrm{Li}$ [2] and in Madden and Stynes [7], but are of different type than the one considered here.

Recently, simple upwinding on a properly chosen Shishkin mesh was analyzed independently in $[5,9]$. Both papers are based on similar estimates for a priori bounds for derivatives. These estimates are also the basis of our analysis, and because [5] and the present paper were written almost in parallel, we present the estimates in Section 2 ( it turns out that Lemma 2.1 and Lemma 2.2 are almost identical with Lemma 3.1 in [9]).

Simple upwinding results in a first order scheme. It is well known that for a convectiondiffusion problem with $\varepsilon_{2}=1$ the streamline diffusion finite element method (SDFEM) can generate a second order scheme [13]. Therefore in the present paper we shall analyze the SDFEM on a properly chosen Shishkin mesh for the two-parameter problem and prove almost second-order pointwise convergence uniformly with respect to the parameters $\varepsilon_{1}, \varepsilon_{2}$.

Our analysis is absolutely different from the analysis in [13]. While Stynes and Tobiska use the consistency error of the difference scheme generated by the SDFEM, our approach uses mainly finite element tools. This technique was already presented in [10] for a convection-diffusion problem with one small parameter and a point source.

In the literature so far convection-duffusion problems $\left(\varepsilon_{2}=1\right)$ and reaction-diffusion problems $\left(\varepsilon_{2}=0\right)$ are handled separately. Our approach offers a unified treatment of problem (1.1) for all possible classes of subproblems.

Throughout the paper $\mathrm{C}$ will represent a constant independent of $\varepsilon_{1}, \varepsilon_{2}$, and of the mesh. For a continuous function $\mathrm{g}$, the value at a point $x_{i}$ will be denoted by $g_{i}$. Furthermore , $\|\cdot\|_{\infty}$ denotes the maximum norm, while $\|\cdot\|_{L_{1}}$ denotes the $L_{1}$ norm.

\section{Some properties of the continuous problem}

The solution of the reduced problem $c u_{0}=f$, in general, does not satisfy the boundary conditions, so there exist boundary layers both at $x=0$ and at $x=1$. To describe the layers let us introduce the characteristic equation

$$
-\varepsilon_{1} \lambda^{2}(x)+\varepsilon_{2} b(x) \lambda(x)+c(x)=0 .
$$

It has two real solutions $\lambda_{0}(x)<0$ and $\lambda_{1}(x)>0$. Because $\left|\lambda_{0}\right|<\lambda_{1}$, the layer at $x=1$ is stronger than the layer at $x=0$. Let

$$
\mu_{0}=-\max _{x \in[0,1]} \lambda_{0}(x) \quad \text { and } \quad \mu_{1}=\min _{x \in[0,1]} \lambda_{1}(x)
$$

Lemma 2.1. For any $0<p<1$ we have up to the a certain order $q$ that depends on the smoothness of the data

$$
\left|u^{(k)}(x)\right| \leqslant C\left\{1+\mu_{0}^{k} e^{-p \mu_{0} x}+\mu_{1}^{k} e^{-p \mu_{1}(1-x)}\right\} \quad \text { for } \quad 0 \leqslant k \leqslant q .
$$

Proof. We shall prove (2.1) by induction with respect to $k$. For $k=0$ the estimate follows immediately from the maximum principle.

Since

$$
(L u)^{\prime}=L u^{\prime}+\varepsilon_{2} b^{\prime} u^{\prime}+c^{\prime} u,
$$


then by differentiating the original equation $k$ times, we get

$$
L_{k} u^{(k)}:=-\varepsilon_{1} u^{(k+2)}+\varepsilon_{2} b u^{(k+1)}+c_{k} u^{(k)}=g_{k}, \quad \text { for } \quad k=1, \ldots
$$

where

$$
c_{0}=c, \quad g_{0}=f
$$

and

$$
c_{k}=c+k \varepsilon_{2} b^{\prime}, \quad g_{k}=g_{k-1}^{\prime}-c_{k-1}^{\prime} u^{(k-1)}, \quad \text { for } \quad k=1, \ldots
$$

The $(k+1)^{t h}$ derivative satisfies the equation

$$
L_{k+1} u^{(k+1)}=g_{k+1}
$$

with

$$
\left|g_{k+1}\right| \leqslant C\left\{1+\mu_{0}^{k} e^{-p \mu_{0} x}+\mu_{1}^{k} e^{-p \mu_{1}(1-x)}\right\} .
$$

In the first step of the proof we assume that we have already proved the inequalities

$$
\left|u^{(k+1)}(0)\right| \leqslant C \mu_{0}^{k+1} \quad \text { and } \quad\left|u^{(k+1)}(1)\right| \leqslant C \mu_{1}^{k+1} .
$$

We shall bound $u^{(k+1)}$ using (2.2), (2.3) and the following barrier function

$$
\Phi=C\left(1+\mu_{0}^{k+1} w_{0, p}(x)+\mu_{1}^{k+1} w_{1, p}(x)\right)
$$

with

$$
w_{0, p}(x)=e^{-p \mu_{0} x} \quad \text { and } \quad w_{1, p}(x)=e^{-p \mu_{1}(1-x)} .
$$

It is sufficient to show that $L_{k+1} w_{0, p} \geqslant \alpha_{0} w_{0, p}$ and $L_{k+1} w_{1, p} \geqslant \alpha_{1} w_{1, p}$ with some $\alpha_{0}, \alpha_{1}>0$. Since

$$
\begin{aligned}
L_{k+1} w_{0, p} & =\left\{-\varepsilon_{1} p^{2} \mu_{0}^{2}-\varepsilon_{2} p \mu_{0} b+c+\mathcal{O}\left(\varepsilon_{2}\right)\right\} w_{0, p} \\
& \geqslant\left\{p\left(-\varepsilon_{1} \mu_{0}^{2}-\varepsilon_{2} b \mu_{0}\right)+c+\mathcal{O}\left(\varepsilon_{2}\right)\right\} w_{0, p} \\
& \geqslant\left\{p\left(-\varepsilon_{1} \lambda_{0}^{2}(x)+\varepsilon_{2} b(x) \lambda_{0}(x)\right)+c(x)+\mathcal{O}\left(\varepsilon_{2}\right)\right\} w_{0, p} \\
& =\left\{c(x)(1-p)+\mathcal{O}\left(\varepsilon_{2}\right)\right\} w_{0, p}
\end{aligned}
$$

for any $0<p<1$, we have the desired property if $\varepsilon_{2}$ is small enough. Similarly, we estimate $L_{k+1} w_{1, p}$.

In the next step we shall prove (2.3) starting from

$$
L_{k} u^{(k)}=g_{k} \text { and }\left|u^{(k)}(0)\right| \leqslant C \mu_{0}^{k}, \quad\left|u^{(k)}(1)\right| \leqslant C \mu_{1}^{k}
$$

Setting

$$
u^{(k)}=y+y_{0} \text { with } y_{0}=u^{(k)}(0)(1-x),
$$

we obtain for $y$ the partially homogeneous problem

$$
\begin{aligned}
& L_{k} y=g_{k}-\varepsilon_{2} b y_{0}^{\prime}-c_{k} y_{0}, \\
& y(0)=0, \quad y(1)=u^{k}(1) .
\end{aligned}
$$

Now we bound $y$ by a barrier function of the type

$$
\Phi=C_{1}\left(1+e^{-p \mu_{0} x}-2 e^{-\theta \mu_{0} x}\right) \mu_{0}^{k}+C_{2}\left(e^{-p \mu_{1}(1-x)}-e^{-p \mu_{1}}\right) \mu_{1}^{k},
$$


where $\theta>0$ is large enough. It is easy to check that $\Phi(0)=0$ and $\Phi(1) \geqslant y(1)$ if $C_{2}$ is sufficiently large. Since $L_{k} e^{-\theta \mu_{0} x} \leqslant 0$ and $L_{k} e^{-p \mu_{0} x} \geqslant 0, L_{k} e^{-p \mu_{1}(1-x)} \geqslant 0$, if $\theta$ and $p$ are chosen adequately, $\Phi$ majorizes $y$ if $C_{1}$ is sufficiently large.

Then the definition of the derivative gives

$$
\left|y^{\prime}(0)\right| \leqslant C \mu_{0}^{k+1}+C \mu_{1}^{k+1} e^{-p \mu_{1}} \leqslant C \mu_{0}^{k+1} .
$$

Similarly we prove the second estimate in (2.3).

From (2.1) we obtain immediately the existence of a Shishkin decomposition [4, p. 892]:

Lemma 2.2. The solution $u$ has the representation $u=S+E_{0}+E_{1}$, where

$$
\left|S^{(k)}(x)\right| \leqslant C \quad \text { for } \quad 0 \leqslant k \leqslant g
$$

and

$$
\begin{array}{ll}
\left|E_{0}^{(k)}(x)\right| \leqslant C \mu_{0}^{k} e^{-p \mu_{0} x} & \text { for } \quad 0 \leqslant k \leqslant g, \\
\left|E_{1}^{(k)}(x)\right| \leqslant C \mu_{1}^{k} e^{-p \mu_{1}(1-x)} & \text { for } \quad 0 \leqslant k \leqslant g .
\end{array}
$$

Let us for a moment assume $b$ and $c$ to be constant. Then the two extremal layer situations are characterized by

$$
\varepsilon_{2}^{2}<<\varepsilon_{1}
$$

which implies $\mu_{0} \approx \mu_{1} \approx \sqrt{c / \varepsilon_{1}}$, and we have layers similar to the reaction-diffusion case $\varepsilon_{2}=0$;

$$
\varepsilon_{1}<<\varepsilon_{2}^{2}
$$

which implies $\mu_{0} \approx c /\left(b \varepsilon_{2}\right)$, but $\mu_{1} \approx\left(b \varepsilon_{2}\right) / \varepsilon_{1}$ is much larger than $\mu_{0}$, so the layer at $x=1$ is much stronger than the layer at $x=0$.

In the intermediate case, when neither $(i)$ nor $(i i)$ hold, $\mu_{0}$ and $\mu_{1}$ move in the interval given by the values from $(i)$ and $(i i)$.

Fig. 1 below presents the exact solutions, for various $\varepsilon_{1}$ and $\varepsilon_{2}$, for the problem considered in $[3]$ :

$$
-\varepsilon_{1} u^{\prime \prime}+\varepsilon_{2} u^{\prime}+u=\cos \pi x \quad x \in(0,1), \quad u(0)=u(1)=0 .
$$
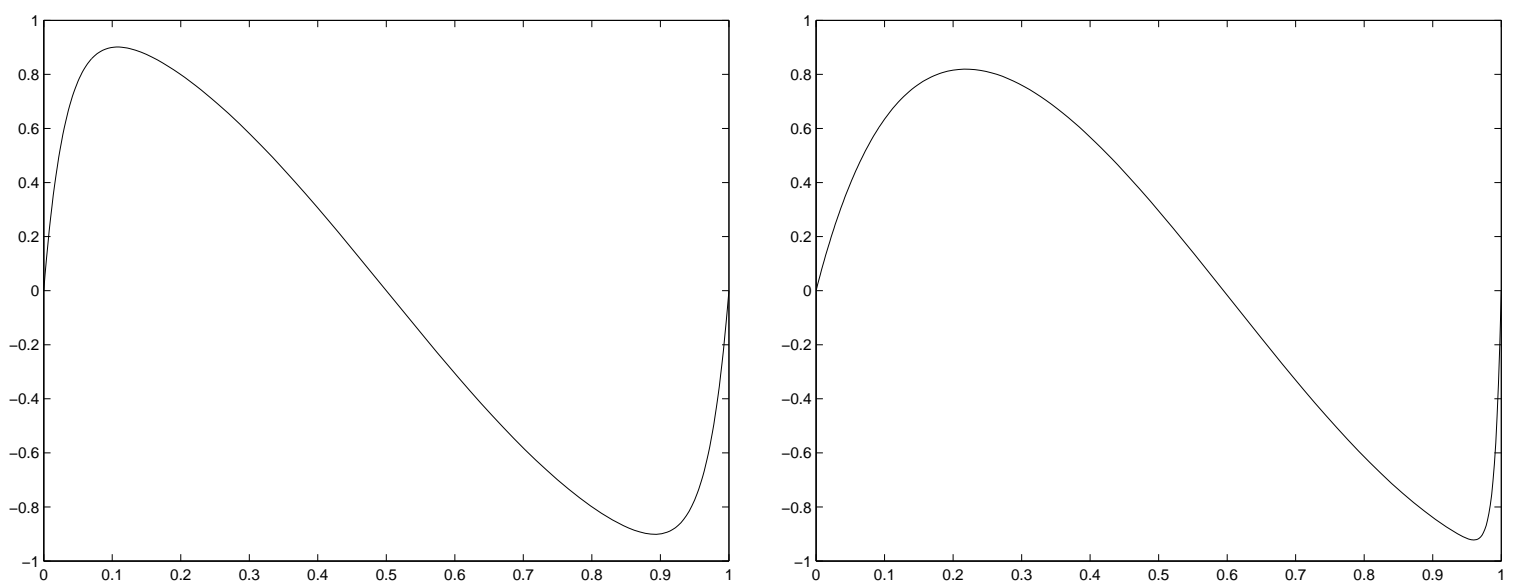

Figure 1. The exact solution for $\varepsilon_{1}=0.001, \varepsilon_{2}=0.01$ and $\varepsilon_{1}=0.001, \varepsilon_{2}=0.1$ 
The exact solution is

$$
u(x)=a \cos \pi x+b \sin \pi x+A e^{\lambda_{0} x}+B e^{-\lambda_{1}(1-x)},
$$

where

$$
\begin{aligned}
a & =\frac{\varepsilon_{1} \pi^{2}+1}{\varepsilon_{2}^{2} \pi^{2}+\left(\varepsilon_{1} \pi^{2}+1\right)^{2}}, & b & =\frac{\varepsilon_{2} \pi}{\varepsilon_{2}^{2} \pi^{2}+\left(\varepsilon_{1} \pi^{2}+1\right)^{2}}, \\
B & =a \frac{1+e^{\lambda_{0}}}{1-e^{\lambda_{0}-\lambda_{1}}}, & \lambda_{0,1} & =\frac{\varepsilon_{2} \mp \sqrt{\varepsilon_{2}^{2}+4 \varepsilon_{1}}}{2 \varepsilon_{1}} .
\end{aligned}
$$

\section{The SDFEM with linear elements}

\subsection{The scheme}

For problem (1.1), the standard weak formulation is: find $u \in V$ such that

$$
a_{G}(u, v):=\left(\varepsilon_{1} u^{\prime}, v^{\prime}\right)+\left(\varepsilon_{2} b u^{\prime}+c u, v\right)=(f, v) \quad \forall v \in V,
$$

where $V=H_{0}^{1}(\Omega)$ denotes the usual Sobolev space and $(\cdot, \cdot)$ is the scalar product on $L_{2}(\Omega)$.

On a given mesh

$$
0=x_{0}<x_{1}<\cdots<x_{N-1}<x_{N}=1 \text {, }
$$

with the mesh step $h_{i}=x_{i}-x_{i-1}, i=1, \ldots, N$, we set $\bar{h}_{i}=\left(h_{i}+h_{i+1}\right) / 2, i=1, \ldots, N-1$, and for a given mesh function $\left\{u_{i}\right\}$ we define the forward and backward difference operators $D^{+}$and $D^{-}$by

$$
D^{+} u_{i}:=\frac{u_{i+1}-u_{i}}{h_{i+1}} \text { and } D^{-} u_{i}:=\frac{u_{i}-u_{i-1}}{h_{i}} .
$$

Let $V_{h} \subset V$ be the space of continuous linear finite elements with the basis functions

$$
\phi_{i}= \begin{cases}\frac{x-x_{i-1}}{h_{i}} & \text { for } x \in\left[x_{i-1}, x_{i}\right], \\ \frac{x_{i+1}-x}{h_{i+1}} & \text { for } x \in\left[x_{i}, x_{i+1}\right], \\ 0 & \text { for } x \notin\left[x_{i-1}, x_{i+1}\right] .\end{cases}
$$

The Galerkin approach is characterized by: find $u_{h} \in V_{h}$ such that

$$
a_{G}\left(u_{h}, v_{h}\right)=\left(f, v_{h}\right) \quad \forall v_{h} \in V_{h} .
$$

Based on the decomposition $u=S+E_{0}+E_{1}$ given in Lemma 2.2, we take $p=1 / 2$ and introduce a corresponding S-mesh. Namely, the mesh is equidistant on $\Omega_{0}:=\left[0, \sigma_{0}\right], \Omega_{g}:=$ $\left[\sigma_{0}, 1-\sigma_{1}\right]$ and $\Omega_{1}:=\left[1-\sigma_{1}, 1\right]$. We choose

$$
\sigma_{0}=\frac{2 \tau}{\mu_{0}} \ln N, \quad \sigma_{1}=\frac{2 \tau}{\mu_{1}} \ln N
$$

(such that $\left.e^{-\mu_{0} x / 2}\right|_{x=\sigma_{0}}=N^{-\tau}$, for example). Since $\mu_{0}$ and $\mu_{1}$ are large and $\mu_{1} \geqslant \mu_{0}$, the mesh is fine on $\Omega_{0}$ and $\Omega_{1}$ and coarse on $\Omega_{g}$, with mesh step sizes

$$
h_{0}=\mathcal{O}\left(\frac{N^{-1} \ln N}{\mu_{0}}\right), \quad h_{1}=\mathcal{O}\left(\frac{N^{-1} \ln N}{\mu_{1}}\right) \quad \text { and } \quad H=h_{g}=\mathcal{O}\left(N^{-1}\right),
$$

respectively.

If $u^{I}$ denotes the linear interpolant of $u$ in $V_{h}$, simple modifications of the well-known techniques of [6] result in: 
Lemma 3.1. The interpolation error satisfies

$$
\left\|u-u^{I}\right\|_{\infty, \Omega_{g}} \leqslant C N^{-2}, \quad\left\|u-u^{I}\right\|_{\infty, \Omega \backslash \Omega_{g}} \leqslant C\left(N^{-1} \ln N\right)^{2} .
$$

The Galerkin method generates a difference scheme which can be written in the form

$$
\begin{aligned}
L_{G}^{N} u_{i} & :=-\varepsilon_{1} \frac{D^{+} u_{i}-D^{-} u_{i}}{\bar{h}_{i}}+\varepsilon_{2}\left(\beta_{i+1} \frac{u_{i+1}-u_{i}}{\bar{h}_{i}}-\beta_{i-1} \frac{u_{i}-u_{i-1}}{\bar{h}_{i}}\right)+\gamma_{i-1} u_{i-1}+\gamma_{i} u_{i}+\gamma_{i+1} u_{i+1} \\
& =\frac{1}{\bar{h}_{i}} \int_{x_{i-1}}^{x_{i+1}} f \phi_{i},
\end{aligned}
$$

where $\phi_{i}$ denotes again the basis function of $V_{h}$ with $\phi_{i}\left(x_{j}\right)=\delta_{i j}$, and

$$
\begin{aligned}
\beta_{i-1} & =\int_{x_{i}-1}^{x_{i}} b \phi_{i-1}^{\prime} \phi_{i}<0, & \beta_{i+1} & =\int_{x_{i}}^{x_{i+1}} b \phi_{i+1}^{\prime} \phi_{i}>0, \\
\gamma_{i-1} & =\frac{1}{\bar{h}_{i}} \int_{x_{i}-1}^{x_{i}} c \phi_{i-1} \phi_{i}, & \gamma_{i} & =\frac{1}{\bar{h}_{i}} \int_{x_{i}-1}^{x_{i+1}} c \phi_{i} \phi_{i}, \quad \gamma_{i+1}=\frac{1}{\bar{h}_{i}} \int_{x_{i}}^{x_{i+1}} c \phi_{i+1} \phi_{i}>0 .
\end{aligned}
$$

When $c=$ const we have $\gamma_{i-1}=h_{i} /\left(6 \bar{h}_{i}\right), \gamma_{i}=2 / 3, \gamma_{i+1}=h_{i+1} /\left(6 \bar{h}_{i}\right)$.

If we wish to have an $M$-matrix, we have the conditions

$$
\varepsilon_{2} \beta_{i+1}+\bar{h}_{i} \gamma_{i+1} \leqslant \frac{\varepsilon_{1}}{h_{i+1}} \quad \text { and } \quad \gamma_{i-1} \bar{h}_{i} \leqslant \varepsilon_{2}\left(-\beta_{i-1}\right)+\frac{\varepsilon_{1}}{h_{i}}
$$

which for a lumped scheme $\left(\gamma_{i-1}=\gamma_{i+1}=0\right.$ and $\left.\gamma_{i}^{\text {new }}=\gamma_{i-1}+\gamma_{i}+\gamma_{i+1}\right)$ reduce to

$$
\varepsilon_{2} \beta_{i+1} \leqslant \frac{\varepsilon_{1}}{h_{i+1}}
$$

Because these conditions are too restrictive, one has to stabilize the FEM to generate $M$ matrices.

For the SDFEM we modify the Galerkin bilinear operator $a_{G}$ by introducing lumping for the $c$ - term:

$$
a_{G L}(u, v):=\varepsilon_{1}\left(u^{\prime}, v^{\prime}\right)+\varepsilon_{2}\left(b u^{\prime}, v\right)+\sum_{k=1}^{N-1} \bar{h}_{k} c_{k} u_{k} v_{k} .
$$

The streamline-diffusion method is characterized by the stabilization

$$
a_{S T}(u, v):=\sum_{k=1}^{N} \int_{x_{k-1}}^{x_{k}} \delta_{k}\left(-\varepsilon_{1} u^{\prime \prime}+\varepsilon_{2} b u^{\prime}+c u\right) v^{\prime}
$$

with some elementwise-defined parameter $\delta_{k}$ which will be determined later.

Now we again choose $V_{h} \subset V$ to be the space of piecewise linear elements on the given grid. Introducing $a(u, v)=a_{G L}(u, v)+a_{S T}(u, v)$, we define the SDFEM discretization: find $u_{h} \in V_{h}$ such that

$$
a\left(u_{h}, v_{h}\right)=f_{h}\left(v_{h}\right) \quad \forall v_{h} \in V_{h}
$$


with

$$
f_{h}\left(v_{h}\right):=\left(f, v_{h}\right)+\sum_{k=1}^{N} \int_{x_{k-1}}^{x_{k}} \delta_{k} f v_{h}^{\prime} .
$$

The SDFEM generates a finite difference scheme, which can be written in the form

$$
L^{N} u_{i}:=-\varepsilon_{1}\left(D^{+} u_{i}-D^{-} u_{i}\right)+\alpha_{i} D^{+} u_{i}+\beta_{i} D^{-} u_{i}+\gamma_{i} u_{i}=f_{h}\left(\varphi_{i}\right),
$$

where

$$
\begin{aligned}
& \alpha_{i}=h_{i+1} \int_{x_{i}}^{x_{i+1}}\left(\varepsilon_{2} b \varphi_{i+1}^{\prime} \varphi_{i}+\delta_{i+1} \varepsilon_{2} b \varphi_{i+1}^{\prime} \varphi_{i}^{\prime}+\delta_{i+1} c \varphi_{i+1} \varphi_{i}^{\prime}\right), \\
& \beta_{i}=h_{i} \int_{x_{i-1}}^{x_{i}}\left(\varepsilon_{2} b \varphi_{i}^{\prime} \varphi_{i}+\delta_{i} \varepsilon_{2} b \varphi_{i}^{\prime} \varphi_{i}^{\prime}+\delta_{i} c \varphi_{i-1} \varphi_{i-1}^{\prime}\right), \\
& \gamma_{i}=\bar{h}_{i} c_{i}+\delta_{i} \int_{x_{i-1}}^{x_{i}} c \varphi_{i}^{\prime}+\delta_{i+1} \int_{x_{i}}^{x_{i+1}} c \varphi_{i}^{\prime} .
\end{aligned}
$$

The choice of the parameter $\delta_{i}$ is determined by the structure of the coefficient matrix of scheme (3.1), as we wish to have an $M$-matrix. Namely, if the local mesh size is small enough, standard Galerkin can be applied,i.e., it is possible to choose $\delta_{i}=0, i=1, \ldots, N$. This leads to the condition

$$
\left.\alpha_{i-1}\right|_{\delta_{i}=0} \leqslant \varepsilon_{1} \quad \text { i.e., } \quad \varepsilon_{2} h_{i} \hat{b}_{i} \leqslant \varepsilon_{1} \text { for } i=1, \ldots, N
$$

where $\hat{b}_{i}=\left(1 / h_{i}\right) \int_{x_{i-1}}^{x_{i}} b \varphi_{i-1}$. Otherwise, we choose $\delta_{i}$ from the condition $\alpha_{i-1}=0, i=$ $1, \ldots, N$. With the notations

$$
\hat{c}_{i}=\frac{1}{h_{i}} \int_{x_{i-1}}^{x_{i}} c \varphi_{i}, \quad \tilde{b}_{i}=\frac{1}{h_{i}} \int_{x_{i}-1}^{x_{i}} b,
$$

this condition is equivalent to

$$
\delta_{i}\left(\frac{\varepsilon_{2} \tilde{b}_{i}}{h_{i}}+\hat{c}_{i}\right)=\varepsilon_{2} \hat{b}_{i}
$$

Finally we obtain

$$
\delta_{i}= \begin{cases}0 & \text { if } \varepsilon_{2} h_{i} \hat{b}_{i} \leqslant \varepsilon_{1} \\ \frac{\varepsilon_{2} \hat{b}_{i} h_{i}}{\varepsilon_{2} \tilde{b}_{i}+\hat{c}_{i} h_{i}} & \text { otherwise. }\end{cases}
$$

We can see that

$$
\begin{aligned}
& \delta_{i}=\mathcal{O}\left(h_{i}\right) \quad \text { if } h_{i} \text { is smaller than } \varepsilon_{2}, \\
& \delta_{i}=\mathcal{O}\left(\varepsilon_{2}\right) \quad \text { if } \varepsilon_{2} \text { is smaller than } h_{i} .
\end{aligned}
$$

In the case of $\varepsilon_{2}=1$ and $\varepsilon_{1}=\varepsilon$, we choose $\delta_{i}=0$ on the fine mesh. If we check the condition $\varepsilon_{2} h_{i} \hat{b}_{i} \leqslant \varepsilon_{1}$, we see that it is satisfied on $\Omega_{1}$ because $\mu_{1} \geqslant\left(\varepsilon_{2} b_{0}\right) / \varepsilon_{1}$, i.e., $\varepsilon_{1} \geqslant \varepsilon_{2} b_{0} / \mu_{1}$. On $\Omega_{0}$, however, the situation is different. 
Summarizing, we have on $\Omega_{1}$ :

$$
\delta_{i}=0
$$

on $\Omega_{g}$ :

$$
\delta_{i}= \begin{cases}0 & \text { if } \varepsilon_{2} H \hat{b}_{i} \leqslant \varepsilon_{1} \\ \frac{\varepsilon_{2} H \hat{b}_{i}}{\varepsilon_{2} \tilde{b}_{i}+\hat{c}_{i} H} & \text { otherwise }\end{cases}
$$

on $\Omega_{0}$ :

$$
\delta_{i}= \begin{cases}0 & \text { if } \varepsilon_{2} h_{0} \hat{b}_{i} \leqslant \varepsilon_{1}, \\ \frac{\varepsilon_{2} h_{0} \hat{b}_{i}}{\varepsilon_{2} \tilde{b}_{i}+\hat{c}_{i} h_{0}} & \text { otherwise. }\end{cases}
$$

Since $h_{0}=\mathcal{O}\left(N^{-1} \ln N / \mu_{0}\right)$ and $1 / \mu_{0}=\mathcal{O}\left(\varepsilon_{2}+\varepsilon_{1}^{1 / 2}\right)$, the stabilization on $\Omega_{0}$ is necessary if $\varepsilon_{1}$ is extremely small, roughly speaking, in comparison with $\varepsilon_{2}^{2} N^{-1} \ln N$. The corresponding stabilization parameter is of order $\mathcal{O}\left(h_{0}\right)$.

\subsection{Pointwise error analysis}

For estimating the pointwise error we use an approach similar to $[10,11]$ based on a discrete Green's function. Let us define a function $G^{j} \in V_{h}$ by

$$
a\left(\varphi_{i}, G^{j}\right)=\delta_{i j} \quad \text { for all } \quad i=1, \ldots, N-1 .
$$

In our estimates we need bounds for the $L_{\infty}$ and $W^{1,1}$ norm of $G^{j}$ (the $L_{1}$ norm of its derivative). Since $G^{j}$ is piecewise linear, the continuous and the discrete versions of these norms are equivalent. For the discrete norms Andreev [1] proved estimates for an upwind finite difference operator in the case of $\varepsilon_{2}=1$. In the Appendix we shall prove similar estimates for the two-parameter problem.

It is a well known fact that the scheme generated by SDFEM can be written as a generalized upwind scheme (see [13] and especially [11], Section 5). We do not want to repeat the details from [11], but it is not difficult to see that scheme (3.1) with (3.2) is equivalent to

$$
L_{h} u_{h}=f_{h}
$$

with

$$
\left(L_{h} u\right)_{i}:=-\frac{\varepsilon_{1}}{\bar{h}_{i}}\left(p_{i+1} D^{+} u_{i}-p_{i} D^{-} u_{i}\right)+\varepsilon_{2} b_{i}^{*} \frac{u_{i}-u_{i-1}}{\bar{h}_{i}}+c_{i}^{*} u_{i} .
$$

Here all coefficients are the result of rewriting (3.1) with (3.2) in this form, especially

$$
c_{i}^{*}=c_{i}+\frac{\delta_{i}}{\bar{h}_{i}} \hat{c}_{i}-\frac{\delta_{i+1}}{\bar{h}_{i}} \hat{c}_{i+1} .
$$

Do we have $c_{i}^{*} \geqslant c_{0}^{*}>0$ if $c_{i} \geqslant c_{0}>0$ ? It is not difficult to see that we are in trouble only if $\delta_{i}=0$ and $\delta_{i+1} \neq 0$, but this happens only in the first subinterval of $\Omega_{g}$ with follows the transition point $\sigma_{0}$. On that interval we have

$$
\delta_{i+1}=\frac{\varepsilon_{2} H \hat{b}_{i}}{\varepsilon_{2} \tilde{b}_{i}+\hat{c}_{i} H} \quad \text { if } \quad \varepsilon_{1} \leqslant \varepsilon_{2} H \hat{b}_{i}
$$


Do we need $c_{i}^{*} \geqslant c_{0}^{*}>0$ ? The proof in the Appendix shows that the strong positivity is only necessary if $\varepsilon_{2}^{2} \leqslant \kappa \varepsilon_{1}$. Let us assume for a moment that $b=$ const. and $c=$ const. Then

$$
c_{i}^{*} \geqslant c\left(1-\frac{2}{H} \frac{\varepsilon_{2}(H / 2) b}{\left(\varepsilon_{2} b+c H / 2\right)}\right)=c \frac{H / 2}{\varepsilon_{2} b+c H / 2} .
$$

Because $\varepsilon_{2}^{2} / \kappa \leqslant \varepsilon_{1} \leqslant \varepsilon_{2} H b$ implies $\varepsilon_{2} \leqslant \kappa b H$, it is true that

$$
\frac{H / 2}{\varepsilon_{2} b+c H / 2} \geqslant \frac{1 / 2}{\kappa b^{2}+c / 2}>0 .
$$

The nonconstant-coefficient case can be handled similarly.

The discrete Green's function $G^{j}$ now satisfies (see the Appendix for some details and estimates for the Green's function)

$$
L_{h} G=\delta_{h}
$$

Using the linear operator $P: V \rightarrow V_{h}$ with

$$
P v:=\sum_{i=1}^{N-1} a\left(v, G^{i}\right) \varphi_{i}
$$

we have the following representation for the error at the mesh point $x_{j}$ :

$$
\left(u-u_{h}\right)\left(x_{j}\right)=\left.P\left(u^{I}-u\right)\right|_{x=x_{j}}+\left.\left(P u-u_{h}\right)\right|_{x=x_{j}}
$$

Furthermore,

$$
\left.P\left(u^{I}-u\right)\right|_{x=x_{j}}=a\left(u-u^{I}, G^{j}\right)
$$

and the consistency error $P u-u_{h}$ vanishes for a consistent finite element method.

Based on the error representation (3.3) we want to estimate the pointwise error of our SDFEM on a S-mesh. Since the stabilization itself is consistent, the consistency error $\left.\left(P u-u_{h}\right)\right|_{x=x_{j}}$ reduces to the error induced by lumping of the $c$-term. That error part can be estimated as in [11] and it is of order $\left(N^{-1} \ln N\right)^{2}$.

To estimate the other error part we start from

$$
\begin{aligned}
a\left(u-u^{I}, G^{j}\right)= & \varepsilon_{1}\left(\left(u-u^{I}\right)^{\prime},\left(G^{j}\right)^{\prime}\right)+\sum_{k=1}^{N-1} \bar{h}_{k} c_{k}\left(u-u^{I}\right)\left(x_{k}\right) G^{j}\left(x_{k}\right) \\
& +\varepsilon_{2}\left(b\left(u-u^{I}\right)^{\prime}, G^{j}\right)+\sum_{k=1}^{N} \delta_{k}\left(-\varepsilon_{1} u^{\prime \prime}+\varepsilon_{2} b\left(u-u^{I}\right)^{\prime}+c\left(u-u^{I}\right),\left(G^{j}\right)^{\prime}\right) .
\end{aligned}
$$

The first two terms are equal to zero. To estimate the remaining terms we want to apply the results concerning the discrete Green's function from the Appendix.

Now we estimate several error contributions. First, using integration by parts

$$
\left|\varepsilon_{2}\left(b\left(u-u^{I}\right)^{\prime}, G^{j}\right)\right| \leqslant C\left\|u-u^{I}\right\|_{\infty}\left\|G^{j}\right\|_{L_{1}}+C\left|\varepsilon_{2}\left(u-u^{I},\left(G^{j}\right)^{\prime}\right)\right| .
$$

Furthermore,

$$
\left|\varepsilon_{2}\left(u-u^{I},\left(G^{j}\right)^{\prime}\right)\right| \leqslant C \varepsilon_{2}\left\|u-u^{I}\right\|_{\infty}\left\|\left(G^{j}\right)^{\prime}\right\|_{L_{1}} \leqslant C \frac{\varepsilon_{2}}{\sqrt{\varepsilon_{2}^{2}+\varepsilon_{1}}}\left(N^{-1} \ln N\right)^{2} .
$$


Next we estimate the contribution of the stabilization on $\Omega_{g}$. Let us recall that

$$
\delta_{g}=\mathcal{O}\left(\frac{\varepsilon_{2} H}{\varepsilon_{2}+H}\right) \quad \text { if } \quad \varepsilon_{1} \leqslant C \varepsilon_{2} H .
$$

(i) $\sum \varepsilon_{1} \delta_{k}\left(u^{\prime \prime},\left(G^{j}\right)^{\prime}\right)$.

For the smooth part we get

$$
C \varepsilon_{1} \delta_{g} \frac{1}{\sqrt{\varepsilon_{2}^{2}+\varepsilon_{1}}} \leqslant C H^{2}, \quad\left(\varepsilon_{1} \leqslant C \varepsilon_{2} H, \quad \delta_{g}=\mathcal{O}(H), \quad \frac{\varepsilon_{2}}{\sqrt{\varepsilon_{2}^{2}+\varepsilon_{1}}} \leqslant 1\right) .
$$

For the layer part we get ( only for $E_{1}$ because that layer is stronger)

$$
C \varepsilon_{1} \delta_{g}\left\|E_{1}^{\prime \prime}\right\|_{L_{1}}\left\|\left(G^{j}\right)^{\prime}\right\|_{\infty} \leqslant C \varepsilon_{1} \delta_{g} \mu_{1} H^{\tau} H^{-1}\left\|G^{j}\right\|_{\infty} .
$$

This error contribution is small enough because $\varepsilon_{1} \mu_{1} / \sqrt{\varepsilon_{2}^{2}+\varepsilon_{1}}$ is bounded.

(ii) $\Sigma \varepsilon_{2} \delta_{k}\left(b\left(u-u^{I}\right)^{\prime},\left(G^{j}\right)^{\prime}\right)$.

Since $\left(G^{j}\right)^{\prime \prime}=0,\left(u-u^{I}\right)_{x_{j}}=0$, integration by parts yields

$$
\left|\Sigma \varepsilon_{2} \delta_{k}\left(b\left(u-u^{I}\right)^{\prime},\left(G^{j}\right)^{\prime}\right)\right| \leqslant C \varepsilon_{2} \delta_{g}\left\|u-u^{I}\right\|_{\infty}\left\|\left(G^{j}\right)^{\prime}\right\|_{L_{1}} \leqslant C \delta_{g} N^{-2} .
$$

(iii) $\quad \Sigma \delta_{k}\left(c\left(u-u^{I}\right),\left(G^{j}\right)^{\prime}\right)$.

Using $\delta_{g}=\mathcal{O}\left(\varepsilon_{2}\right)$, we get

$$
\left|\Sigma \delta_{k}\left(c\left(u-u^{I}\right),\left(G^{j}\right)^{\prime}\right)\right| \leqslant C \varepsilon_{2} \frac{1}{\sqrt{\varepsilon_{2}^{2}+\varepsilon_{1}}}\left\|u-u^{I}\right\|_{\infty} \leqslant C N^{-2} .
$$

Finally, we consider the contributions of the stabilization on $\Omega_{0}$. Let us recall that

$$
\delta_{0}=\mathcal{O}\left(h_{0}\right) \quad \text { if } \quad \varepsilon_{1} \leqslant C \varepsilon_{2} h_{0},
$$

where

$$
h_{0}=\mathcal{O}\left(\frac{N^{-1} \ln N}{\mu_{0}}\right), \quad \frac{1}{\mu_{0}}=\mathcal{O}\left(\varepsilon_{2}+\sqrt{\varepsilon_{2}^{2}+\varepsilon_{1}}\right) .
$$

(i') $\quad \Sigma \varepsilon_{1} \delta_{0}\left(u^{\prime \prime},\left(G^{j}\right)^{\prime}\right)$.

For the smooth part there is no problem. For the layer part $E_{2}\left(E_{1}\right.$ is extremely small on $\left.\Omega_{0}\right)$ using $\varepsilon_{1} \leqslant C \varepsilon_{2} h_{0}, h_{0}=\mathcal{O}\left(N^{-1} \ln N\right) / \mu_{0}$, we get

$$
\left|\Sigma \varepsilon_{1} \delta_{0}\left(u^{\prime \prime},\left(G^{j}\right)^{\prime}\right)\right| \leqslant \varepsilon_{1} \delta_{0} \mu_{0}^{2}\left\|\left(G^{j}\right)^{\prime}\right\|_{L_{1}} \leqslant C \varepsilon_{2}\left(h_{0} \mu_{0}\right)^{2} \frac{1}{\sqrt{\varepsilon_{2}^{2}+\varepsilon_{1}}} \leqslant C\left(N^{-1} \ln N\right)^{2} .
$$

The two other error terms on $\Omega_{0}$, which correspond to (ii) and (iii), can be estimated as on $\Omega_{g}$.

Theorem 3.1. The pointwise error of the SDFEM on a S-mesh satisfies the following estimate:

$$
\left\|u-u_{h}\right\|_{\infty} \leqslant C\left(N^{-1} \ln N\right)^{2}
$$




\section{Appendix: The discrete Green's function}

Denoting the upwind difference operator on an arbitrary mesh by $L_{h}$ :

$$
\left(L_{h} u_{i}\right):=-\frac{\varepsilon_{1}}{\bar{h}_{i}}\left(D^{+} u_{i}-D^{-} u_{i}\right)+b_{i} \varepsilon_{2} \frac{u_{i}-u_{i-1}}{\bar{h}_{i}}+c_{i} u_{i},
$$

(assuming $b_{i}>0, c_{i}>0$ as above), we define the discrete Green's function $G\left(x_{i}, \xi_{j}\right)$ for $L_{h}$, for fixed $\xi_{j}$, by

$$
\begin{gathered}
L_{h} G\left(x_{i}, \xi_{j}\right)=\delta_{h}\left(x_{i}, \xi_{j}\right) \quad G\left(0, \xi_{j}\right)=G\left(1, \xi_{j}\right)=0, \\
\delta_{h}\left(x_{i}, \xi_{j}\right)= \begin{cases}\frac{1}{\bar{h}_{i}} & \text { if } \quad i=j, \\
0 & \text { if } i \neq j .\end{cases}
\end{gathered}
$$

Setting $W=\sqrt{\varepsilon_{2}^{2}+\varepsilon_{1}}$, we shall prove

$$
\left\|G\left(x_{i}, \xi_{j}\right)\right\|_{\infty} \leqslant \frac{C}{W}
$$

To do this, let us introduce the barrier function $w$ defined by

$$
w_{j}=\frac{C}{W}\left\{\begin{array}{l}
\prod_{k=i}^{j}\left(1-\frac{\mu_{0}}{2} h_{k}\right)^{-1} \quad \text { for } \quad j>i, \\
\prod_{k=j}^{i}\left(1+\frac{\mu_{1}}{2} h_{k}\right)^{-1} \text { for } j<i .
\end{array}\right.
$$

From [3] we know already that $L_{h} w \geqslant 0$ at the mesh points $\left\{x_{1}, \ldots, x_{i-1}\right\}$ and $\left\{x_{i+1}, \ldots, x_{N-1}\right\}$. It remains to verify

$$
\left(L_{h} w\right)_{i} \geqslant \frac{1}{\bar{h}_{i}}
$$

if the constant $C$ in (4.2) is chosen adequately.

We use the abbreviations

$$
\bar{\mu}_{0}=\frac{\mu_{0}}{2}<0, \quad \bar{\mu}_{1}=\frac{\mu_{1}}{2}>0
$$

and introduce

$$
\tilde{w}=\left(\begin{array}{l}
\tilde{w}_{i+1} \\
\tilde{w}_{i} \\
\tilde{w}_{i-1}
\end{array}\right)=\left(\begin{array}{l}
\left(1-\bar{\mu}_{0} h_{i+1}\right)^{-1} \\
1 \\
\left(1+\bar{\mu}_{1} h_{i}\right)^{-1}
\end{array}\right)
$$

Then

$$
D^{+} \tilde{w}=\frac{\bar{\mu}_{0}}{1-\bar{\mu}_{0} h_{i+1}}, \quad D^{-} \tilde{w}=\frac{\bar{\mu}_{1}}{1+\bar{\mu}_{1} h_{i}},
$$

and we obtain

$$
\left(L_{h} w_{i}\right)_{i}=\frac{C}{W}\left(-\frac{\varepsilon_{1}}{\bar{h}_{i}}\left(\frac{\bar{\mu}_{0}}{1-\bar{\mu}_{0} h_{i+1}}-\frac{\bar{\mu}_{1}}{1+\bar{\mu}_{1} h_{i}}\right)+\varepsilon_{2} b_{i} h_{i} \frac{\bar{\mu}_{1}}{\bar{h}_{i}\left(1+\bar{\mu}_{1} h_{i}\right)}+c_{i}\right)
$$


Case $A: \varepsilon_{2}^{2} \leqslant \kappa \varepsilon_{1}$.

In this case we neglect the $b$-term and use the existence of a constant $C_{1}$ such that

$$
\mu_{1} \leqslant 2 C_{1} \frac{\varepsilon_{2}+W}{\varepsilon_{1}}=2 \mu_{1}^{*} \quad \text { as well as } \quad-\mu_{0} \leqslant 2 \mu_{1}^{*}
$$

Then

$$
\frac{1}{1-\bar{\mu}_{0} h_{i+1}} \geqslant \frac{1}{1+\mu_{1}^{*} \bar{h}_{i}}, \quad \frac{1}{1+\bar{\mu}_{1} h_{i}} \geqslant \frac{1}{1+\mu_{1}^{*} \bar{h}_{i}}
$$

and we obtain

$$
\left(L_{h} w_{i}\right)_{i} \geqslant \frac{C}{\bar{h}_{i}}\left(\frac{\varepsilon_{1}\left(\bar{\mu}_{1}-\bar{\mu}_{0}\right)}{W\left(1+\mu_{1}^{*} \bar{h}_{i}\right)}+c_{i} \frac{\bar{h}_{i}}{W}\right) .
$$

If $\bar{h}_{i} \geqslant \rho W$ for some $\rho>0$, we have the desired estimate. Otherwise, we have

$$
\frac{\varepsilon_{1}\left(\bar{\mu}_{1}-\bar{\mu}_{0}\right)}{W\left(1+\mu_{1}^{*} \bar{h}_{i}\right)} \geqslant \frac{\varepsilon_{1}\left(\bar{\mu}_{1}-\bar{\mu}_{0}\right)}{W} \frac{1}{1+\mu_{1}^{*} \rho \bar{h}_{i}} .
$$

Since $\varepsilon_{1}\left(\bar{\mu}_{1}-\bar{\mu}_{0}\right) / W$ is bounded away from zero and $\mu_{1}^{*} W$ is bounded if $\varepsilon_{2}^{2} \leqslant \kappa \varepsilon_{1}$, it follows that (4.3) holds true if $\mathrm{C}$ is chosen adequately.

Case $B: \varepsilon_{2}^{2} \geqslant \kappa \varepsilon_{1}$

In this case we neglect the $c$-term and get

$$
\begin{aligned}
\left(L_{h} w\right)_{i} & \geqslant \frac{C}{W} \quad \frac{1}{\bar{h}_{i}\left(1+\bar{\mu}_{1} h_{i}\right)} \quad\left\{\varepsilon_{1} \bar{\mu}_{1}-\varepsilon_{1} \bar{\mu}_{0} \frac{1+\bar{\mu}_{1} h_{i}}{1-\bar{\mu}_{0} h_{i+1}}+\varepsilon_{2} b_{i} \bar{\mu}_{1} h_{i}\right\} \\
& =\frac{C}{W} \quad \frac{1}{\bar{h}_{i}\left(1+\bar{\mu}_{1} h_{i}\right)} \quad\left\{\varepsilon_{1}\left(\bar{\mu}_{1}-\bar{\mu}_{0}\right)-\varepsilon_{1} \bar{\mu}_{0} \frac{\bar{\mu}_{1} h_{i}+\bar{\mu}_{0} h_{i+1}}{1-\bar{\mu}_{0} h_{i+1}}+\varepsilon_{2} b_{i} \bar{\mu}_{1} h_{i}\right\} .
\end{aligned}
$$

Using

$$
\bar{\mu}_{1} h_{i}+\bar{\mu}_{0} h_{i+1}=\bar{\mu}_{1} h_{i}\left(1-\bar{\mu}_{0} h_{i+1}\right)+\bar{\mu}_{0} h_{i+1}\left(1+\bar{\mu}_{1} h_{i}\right)
$$

we obtain

$$
\begin{aligned}
\left(L_{h} w\right)_{i} & \geqslant \frac{C}{W} \frac{1}{\bar{h}_{i}\left(1+\bar{\mu}_{1} h_{i}\right)}\left\{\varepsilon_{1}\left(\bar{\mu}_{1}-\bar{\mu}_{0}\right)+\left(\varepsilon_{2} b_{i}-\varepsilon_{1} \bar{\mu}_{0}\right) \bar{\mu}_{1} h_{i}-\frac{\varepsilon_{1} \bar{\mu}_{0} \bar{\mu}_{0} h_{i+1}\left(1+\bar{\mu}_{1} h_{i}\right)}{1-\bar{\mu}_{0} h_{i+1}}\right\} \\
& =\frac{C}{\bar{h}_{i}}\left\{\frac{\frac{\varepsilon_{1}\left(\bar{\mu}_{1}-\bar{\mu}_{0}\right)}{W}+\frac{\varepsilon_{2} b_{i}-\varepsilon_{1} \bar{\mu}_{0}}{W} \bar{\mu}_{1} h_{i}}{1+\bar{\mu}_{1} h_{i}}-\frac{\varepsilon_{1} \bar{\mu}_{0} \bar{\mu}_{0} h_{i+1}}{W\left(1-\bar{\mu}_{0} h_{i+1}\right)}\right\} .
\end{aligned}
$$

This expression has the structure

$$
\left(L_{h} w\right)_{i} \geqslant \frac{C}{W}\left\{\frac{D+E \bar{\mu}_{1} h_{i}}{1+\bar{\mu}_{1} h_{i}}-F\right\}
$$

with $|F|<E,|F|<D$ and $|F|<-\varepsilon_{1} \bar{\mu}_{0} / W$.

Since $D-F$ is bounded away from zero, it remains to show that $E-F$ is bounded away from zero also. We have the necessary bound since $\varepsilon_{2}^{2} \geqslant \kappa \varepsilon_{1}$ and $E-F>\varepsilon_{2} b_{i} / W$. 
Remark 4.1. It is a simple exercise to replace the difference operator by the more general operator

$$
\left(L_{h} u\right)_{i}:=-\frac{\varepsilon_{1}}{\bar{h}_{i}}\left(p_{i+1} D^{+} u_{i}-p_{i} D^{-} u_{i}\right)+\varepsilon_{2} b_{i}^{*} \frac{u_{i}-u_{i-1}}{\bar{h}_{i}}+c_{i}^{*} u_{i}
$$

assuming $p_{i} \geqslant p>0$, and again $b_{i}^{*}>0, c_{i}^{*}>0$. (SDFEM is related to a difference operator of that form)

Remark 4.2. The estimate remains true if we consider the standard upwind difference operator with the difference quotient $\left(u_{i}-u_{i-1}\right) / h_{i}$.

Let us summarize the properties of the discrete Green's function for the two parameter problem:

Lemma 4.1. The discrete Green's function G satisfies

$$
\|G\|_{L_{1}} \leqslant C ; \quad\|G\|_{\infty} \leqslant \frac{C}{\sqrt{\varepsilon_{2}^{2}+\varepsilon_{1}}} ; \quad\left\|G^{\prime}\right\|_{L_{1}} \leqslant \frac{C}{\sqrt{\varepsilon_{2}^{2}+\varepsilon_{1}}} .
$$

The $L_{1}$ estimate can be obtained by summing up the equations for $G$ at all meshpoints. The $W^{1,1}$ bound follows from the $L_{\infty}$ bound from the same arguments used by Andreev [1, p. 925].

\section{Numerical experiments}

In this section we present some numerical results for scheme (3.1), applied to the following example.

\section{Example 5.1.}

$$
-\varepsilon_{1} u^{\prime \prime}+\varepsilon_{2} u^{\prime}+u=\cos \pi x \quad x \in(0,1), \quad u(0)=u(1)=0 .
$$

The exact solution is

$$
u(x)=a \cos \pi x+b \sin \pi x+A e^{\lambda_{0} x}+B e^{-\lambda_{1}(1-x)},
$$

where

$$
\begin{aligned}
a=\frac{\varepsilon_{1} \pi^{2}+1}{\varepsilon_{2}^{2} \pi^{2}+\left(\varepsilon_{1} \pi^{2}+1\right)^{2}}, & b=\frac{\varepsilon_{2} \pi}{\varepsilon_{2}^{2} \pi^{2}+\left(\varepsilon_{1} \pi^{2}+1\right)^{2}}, \\
A=-a \frac{1+e^{-\lambda_{1}}}{1-e^{\lambda_{0}-\lambda_{1}}}, & B=a \frac{1+e^{\lambda_{0}}}{1-e^{\lambda_{0}-\lambda_{1}}}, \quad \lambda_{0,1}=\frac{\varepsilon_{2} \mp \sqrt{\varepsilon_{2}^{2}+4 \varepsilon_{1}}}{2 \varepsilon_{1}} .
\end{aligned}
$$


Table 1. Errors $E^{N}$ at the mesh points and convergence rates $\operatorname{Ord}^{N}$ for $N=2^{5}$

\begin{tabular}{|c|c|c|c|c|c|c|c|c|}
\hline \multirow[t]{2}{*}{$\varepsilon_{2}$} & \multicolumn{7}{|c|}{$\varepsilon_{1}$} & \\
\hline & 1 & $10^{-2}$ & $10^{-4}$ & $10^{-6}$ & $10^{-8}$ & $10^{-10}$ & $10^{-12}$ & \\
\hline \multirow[t]{2}{*}{1} & $2.272 \mathrm{e}-05$ & $124 \mathrm{e}-04$ & $1.513 \mathrm{e}-03$ & $686 \mathrm{e}-02$ & $.962 \mathrm{e}-02$ & $1.031 \mathrm{e}-01$ & $1.024 \mathrm{e}-01$ & $E^{N}$ \\
\hline & 2.004 & 002 & 2.028 & .782 & -0.033 & & -0.096 & $O r d^{1}$ \\
\hline \multirow[t]{2}{*}{$10^{-1}$} & $1.189 \mathrm{e}-05$ & $316 \mathrm{e}-04$ & $.723 \mathrm{e}-04$ & $.173 \mathrm{e}-02$ & $1.585 \mathrm{e}-01$ & $2.067 \mathrm{e}-01$ & $2.215 \mathrm{e}-01$ & $E^{N}$ \\
\hline & 2.004 & 2.003 & 2.006 & 2.130 & 0.411 & 0.0897 & 0.094 & $O r d^{N}$ \\
\hline \multirow[t]{2}{*}{$10^{-2}$} & $6.416 \mathrm{e}-06$ & $.698 \mathrm{e}-05$ & $4.598 \mathrm{e}-04$ & $4.171 \mathrm{e}-03$ & $2.822 \mathrm{e}-01$ & $3.223 \mathrm{e}-01$ & $3.384 \mathrm{e}-01$ & $E^{N}$ \\
\hline & 2.002 & 2.002 & 2.002 & 2.028 & 3.843 & 0.097 & 0.088 & $O r d^{\Lambda}$ \\
\hline \multirow[t]{2}{*}{$10^{-3}$} & $3.850 \mathrm{e}-06$ & $4.882 \mathrm{e}-05$ & $3.673 \mathrm{e}-04$ & $1.124 \mathrm{e}-03$ & $2.330 \mathrm{e}-02$ & $2.968 \mathrm{e}-01$ & $2.981 \mathrm{e}-01$ & $E^{N}$ \\
\hline & 2.002 & 2.001 & 1.998 & 1.996 & 2.136 & 0.028 & 0.100 & $O r d^{\Lambda}$ \\
\hline \multirow[t]{2}{*}{$10^{-4}$} & $2.682 \mathrm{e}-06$ & $.538 \mathrm{c}$ & $3.068 \mathrm{e}-1$ & 1.268 & $3.597 \mathrm{e}-03$ & 1.772 & 1.882 & $E^{N}$ \\
\hline & & & & & & & & \\
\hline \multirow[t]{2}{*}{$10^{-5}$} & $2.147 \mathrm{e}-06$ & 2.900 & $2.691 \mathrm{e}$ & 1.260 & $4.243 \mathrm{e}-03$ & $1.392 \mathrm{e}$ & $1.352 \mathrm{e}-01$ & $E^{N}$ \\
\hline & 2.00 & & 1.9 & & & & 0.497 & Ord \\
\hline \multirow[t]{2}{*}{$10^{-6}$} & $1.887 \mathrm{e}$ & 2.588 & $2.486 \mathrm{e}$ & 1.22 & 4.255 & 1.467 & $4.869 \mathrm{e}-02$ & $E^{N}$ \\
\hline & 1.996 & & 1.99 & & & & 1.9 & Ord \\
\hline \multirow[t]{2}{*}{$10^{-7}$} & $1.770 \mathrm{e}-06$ & 2.441 & $2.373 \mathrm{e}-04$ & $.189 \mathrm{e}$ & $4.216 \mathrm{e}-03$ & $1.524 \mathrm{e}-0$ & $4.609 \mathrm{e}-02$ & $E^{N}$ \\
\hline & 2.001 & & 1.99 & 1.9 & 1.974 & 1.9 & 1.670 & Ord \\
\hline \multirow[t]{2}{*}{$10^{-8}$} & $1.711 \mathrm{e}-06$ & $2.367 \mathrm{e}-05$ & $2.316 \mathrm{e}-04$ & $1.169 \mathrm{e}-03$ & $4.154 \mathrm{e}-03$ & $1.505 \mathrm{e}-02$ & $4.266 \mathrm{e}-02$ & $E^{N}$ \\
\hline & 2.001 & 2.000 & 1.994 & 988 & 1.978 & 1.912 & 1.506 & Ord \\
\hline \multirow[t]{2}{*}{$10^{-9}$} & $1.682 \mathrm{e}-06$ & $2.329 \mathrm{e}-05$ & $2.290 \mathrm{e}-04$ & $1.158 \mathrm{e}-03$ & $4.112 \mathrm{e}-03$ & $1.480 \mathrm{e}-02$ & $3.760 \mathrm{e}-02$ & $E^{N}$ \\
\hline & 2.000 & 2.000 & 1.995 & 1.987 & 1.978 & 1.912 & 1.344 & Ord \\
\hline \multirow[t]{2}{*}{$10^{-10}$} & $1.667 \mathrm{e}-06$ & $2.311 \mathrm{e}-05$ & $2.277 \mathrm{e}-04$ & $1.153 \mathrm{e}-03$ & $4.088 \mathrm{e}-03$ & $1.463 \mathrm{e}-02$ & $3.494 \mathrm{e}-02$ & $E^{N}$ \\
\hline & 000 & 2.00 & 1.996 & 1.9 & 1.978 & 1.913 & 1.264 & $\mathrm{Ord}^{1}$ \\
\hline \multirow[t]{2}{*}{$10^{-11}$} & $1.656 \mathrm{e}-06$ & $2.296 \mathrm{e}-05$ & $2.267 \mathrm{e}-04$ & 1.150 & $4.068 \mathrm{e}-03$ & $1.449 \mathrm{e}-02$ & $3.290 \mathrm{e}-02$ & $E^{N}$ \\
\hline & 2.000 & 2.000 & 1.996 & 1.987 & 1.978 & 1.91 & 1.204 & Ord \\
\hline \multirow[t]{2}{*}{$10^{-12}$} & $1.653 \mathrm{e}-06$ & $2.293 \mathrm{e}-05$ & $2.265 \mathrm{e}-04$ & 1.149 & $4.063 \mathrm{e}-03$ & $1.4+40-02$ & $3.238 \mathrm{e}-02$ & $E^{N}$ \\
\hline & 2.000 & & & & & & & \\
\hline \multirow[t]{2}{*}{$10^{-13}$} & $1.652 \mathrm{e}-06$ & $2.292 \mathrm{e}-05$ & $2.264 \mathrm{e}-04$ & 1.148 & $4.062 \mathrm{e}-03$ & 1.444 & $3.221 \mathrm{e}-02$ & $E^{N}$ \\
\hline & & & & & & & 1.184 & $\mathrm{Ord}^{2}$ \\
\hline \multirow[t]{2}{*}{$10^{-14}$} & $1.652 \mathrm{e}-06$ & $2.292 \mathrm{e}-05$ & $2.264 \mathrm{e}-04$ & $1.148 \mathrm{e}-03$ & $4.061 \mathrm{e}-03$ & $1.444 \mathrm{e}-02$ & $3.221 \mathrm{e}-02$ & $E^{N}$ \\
\hline & 2.000 & 2.000 & 1.996 & 1.987 & 1.978 & 1.913 & 1.184 & \\
\hline
\end{tabular}

In Table 1 and Table 2 we present maximum pointwise errors $E^{N}$ for $N=2^{5}$ and $N=2^{10}$, respectively, which are given by

$$
E^{N}=\max _{0 \leqslant i \leqslant N}\left|u\left(x_{i}\right)-u_{i}\right|
$$

Assuming the convergence order $\left(N^{-1}\right)^{r}$, for some $r$, we use the double-mesh method to compute the experimental rates of convergence $\operatorname{Ord}^{N}$. For every fixed $\varepsilon_{1}$ and $\varepsilon_{2}$, we computed the rate of convergence from

$$
\operatorname{Ord}^{N}=\frac{\ln E^{N}-\ln E^{2 N}}{\ln 2}
$$

The results clearly show robust uniform convergence of order two. 
Table 2. Errors $E^{N}$ at the mesh points and convergence rates $\operatorname{Ord}^{N}$ for $N=2^{10}$

\begin{tabular}{|c|c|c|c|c|c|c|c|c|}
\hline \multirow[t]{2}{*}{$\varepsilon_{2}$} & \multicolumn{7}{|c|}{$\varepsilon_{1}$} & \\
\hline & 1 & $10^{-2}$ & $10^{-4}$ & $10^{-6}$ & $10^{-8}$ & $10^{-10}$ & $10^{-12}$ & \\
\hline \multirow[t]{2}{*}{1} & $6.768 \mathrm{e}-08$ & $7.393 \mathrm{e}-07$ & $5.712 \mathrm{e}-06$ & $6.753 \mathrm{e}-05$ & $1.502 \mathrm{e}-04$ & $1.407 \mathrm{e}-01$ & $1.063 \mathrm{e}-01$ & $E^{N}$ \\
\hline & 4.996 & 2.577 & 2.037 & 2.003 & 1.700 & 11.58 & -0.002 & $O r d^{N}$ \\
\hline \multirow[t]{2}{*}{$10^{-1}$} & $3.532 \mathrm{e}-08$ & $4.236 \mathrm{e}-07$ & $3.283 \mathrm{e}-06$ & $4.074 \mathrm{e}-05$ & $3.732 \mathrm{e}-04$ & $3.813 \mathrm{e}-04$ & $1.969 \mathrm{e}-01$ & $E^{N}$ \\
\hline & 4.674 & 3.404 & 2.145 & 2.005 & 1.701 & 1.686 & -0.443 & $O r d^{N}$ \\
\hline \multirow[t]{2}{*}{$10^{-2}$} & $1.982 \mathrm{e}-08$ & $2.504 \mathrm{e}-07$ & $1.595 \mathrm{e}-06$ & $1.595 \mathrm{e}-05$ & $2.843 \mathrm{e}-04$ & $7.012 \mathrm{e}-04$ & $3.467 \mathrm{e}-01$ & $E^{N}$ \\
\hline & 3.566 & 3.223 & 2.674 & 2.019 & 2.000 & 1.693 & 10.58 & $O r d^{N}$ \\
\hline \multirow[t]{2}{*}{$10^{-3}$} & $1.268 \mathrm{e}-08$ & $1.680 \mathrm{e}-07$ & $1.338 \mathrm{e}-06$ & $4.249 \mathrm{e}-06$ & $8.206 \mathrm{e}-05$ & $8.452 \mathrm{e}-04$ & $9.594 \mathrm{e}-04$ & $E^{N}$ \\
\hline & 2.905 & 2.683 & 2.351 & 2.167 & 2.002 & 1.698 & 1.691 & $O r d^{N}$ \\
\hline \multirow[t]{2}{*}{$10^{-4}$} & $9.414 \mathrm{e}-09$ & $1.281 \mathrm{e}-07$ & $1.156 \mathrm{e}-06$ & $4.907 \mathrm{e}-06$ & $1.433 \mathrm{e}-05$ & $3.720 \mathrm{e}-04$ & $9.376 \mathrm{e}-04$ & $E^{N}$ \\
\hline & 2.499 & 2.372 & 2.171 & 2.059 & 2.015 & 2.000 & 1.699 & $O r d^{N}$ \\
\hline \multirow[t]{2}{*}{$10^{-5}$} & $7.889 \mathrm{e}-09$ & $1.086 \mathrm{e}-07$ & $1.033 \mathrm{e}-06$ & $4.916 \mathrm{e}-06$ & $1.693 \mathrm{e}-05$ & $5.448 \mathrm{e}-05$ & $6.448 \mathrm{e}-04$ & $E^{N}$ \\
\hline & 2.264 & 2.196 & 2.086 & 2.026 & 2.005 & 2.001 & 1.706 & $O r d^{N}$ \\
\hline \multirow[t]{2}{*}{$10^{-6}$} & $7.158 \mathrm{e}-09$ & $9.900 \mathrm{e}-08$ & $9.630 \mathrm{e}-07$ & $4.782 \mathrm{e}-06$ & $1.724 \mathrm{e}-05$ & $6.425 \mathrm{e}-05$ & $2.149 \mathrm{e}-04$ & $E^{N}$ \\
\hline & 2.136 & 2.101 & 2.044 & 2.013 & 2.002 & 2.000 & 1.999 & $O r d^{N}$ \\
\hline \multirow[t]{2}{*}{$10^{-7}$} & $6.801 \mathrm{e}-09$ & $9.426 \mathrm{e}-08$ & $9.258 \mathrm{e}-07$ & $4.672 \mathrm{e}-06$ & $1.693 \mathrm{e}-05$ & $6.529 \mathrm{e}-05$ & $2.530 \mathrm{e}-04$ & $E^{N}$ \\
\hline & 2.069 & 2.052 & 2.022 & 2.006 & 2.001 & 1.999 & 1.998 & $O r d^{N}$ \\
\hline \multirow[t]{2}{*}{$10^{-8}$} & $6.625 \mathrm{e}-09$ & $9.190 \mathrm{e}-08$ & $9.067 \mathrm{e}-07$ & $4.606 \mathrm{e}-06$ & $1.663 \mathrm{e}-05$ & $6.404 \mathrm{e}-05$ & $2.570 \mathrm{e}-04$ & $E^{N}$ \\
\hline & 2.035 & 2.026 & 2.011 & 2.003 & 2.000 & 1.999 & 1.999 & $\operatorname{Ord}_{u^{n}}$ \\
\hline \multirow[t]{2}{*}{$10^{-9}$} & $6.538 \mathrm{e}-09$ & $9.072 \mathrm{e}-08$ & $8.971 \mathrm{e}-07$ & $4.570 \mathrm{e}-06$ & $1.644 \mathrm{e}-05$ & $6.285 \mathrm{e}-05$ & $2.516 \mathrm{e}-04$ & $E^{N}$ \\
\hline & 2.018 & 2.013 & 2.006 & 2.002 & 2.000 & 2.000 & 1.997 & $O r d^{N}$ \\
\hline \multirow[t]{2}{*}{$10^{-10}$} & $6.494 \mathrm{e}-09$ & $9.013 \mathrm{e}-08$ & $8.922 \mathrm{e}-07$ & $4.551 \mathrm{e}-06$ & $1.634 \mathrm{e}-05$ & $6.207 \mathrm{e}-05$ & $2.470 \mathrm{e}-04$ & $E^{N}$ \\
\hline & 2.009 & 2.007 & 2.003 & 2.001 & 2.000 & 1.999 & 1.998 & $O r d^{N}$ \\
\hline \multirow[t]{2}{*}{$10^{-11}$} & $6.462 \mathrm{e}-09$ & $8.969 \mathrm{e}-08$ & $8.885 \mathrm{e}-07$ & $4.537 \mathrm{e}-06$ & $1.625 \mathrm{e}-05$ & $6.144 \mathrm{e}-05$ & $2.423 \mathrm{e}-04$ & $E^{N}$ \\
\hline & 2.002 & 2.002 & 2.001 & 2.000 & 2.000 & 1.999 & 1.998 & $O r d^{N}$ \\
\hline \multirow[t]{2}{*}{$10^{-12}$} & $6.454 \mathrm{e}-09$ & $8.958 \mathrm{e}-08$ & $8.876 \mathrm{e}-07$ & $4.533 \mathrm{e}-06$ & $1.623 \mathrm{e}-05$ & $6.127 \mathrm{e}-05$ & $2.410 \mathrm{e}-04$ & $E^{N}$ \\
\hline & 2.001 & 2.000 & 2.000 & 2.000 & 2.000 & 1.999 & 1.998 & $\operatorname{Ord}_{u^{n}}$ \\
\hline \multirow[t]{2}{*}{$10^{-13}$} & $6.451 \mathrm{e}-09$ & $8.955 \mathrm{e}-08$ & $8.873 \mathrm{e}-07$ & $4.532 \mathrm{e}-06$ & $1.623 \mathrm{e}-05$ & $6.122 \mathrm{e}-05$ & $2.406 \mathrm{e}-04$ & $E^{N}$ \\
\hline & 2.000 & 2.000 & 2.000 & 2.000 & 2.000 & 1.999 & 1.998 & $O r d^{N}$ \\
\hline \multirow[t]{2}{*}{$10^{-14}$} & $6.451 \mathrm{e}-09$ & $8.955 \mathrm{e}-08$ & $8.873 \mathrm{e}-07$ & $4.532 \mathrm{e}-06$ & $1.623 \mathrm{e}-05$ & $6.121 \mathrm{e}-05$ & $2.406 \mathrm{e}-04$ & $E^{N}$ \\
\hline & 2.000 & 2.000 & 2.000 & 2.000 & 2.000 & 1.999 & 1.998 & $O r d^{N}$ \\
\hline
\end{tabular}

\section{References}

[1] V. B. Andreev, The Green function and a priori estimates of solutions of monotone three-point singularly perturbed finite-difference schemes, Differ. Equations, 37 (2001), pp. 923-933.

[2] J. Li, A robust finite element method for a singularly perturbed elliptic problem with two small parameters, Comput. Math. Appl., 36 (1998), pp. 91-110.

[3] T. Linß, Untersuchungen zur numerischen Behandlung singulär gestörter Randwert- und Anfangsrandwertaufgaben auf Shishkin-Gittern, Diploma thesis, Department of Math., Technical University of Dresden, 1996.

[4] T. Linß, The necessity of Shishkin decompositions, Appl. Math. Lett., 14 (2001), pp. 891-896.

[5] T. Linß and H.-G. Roos, Analysis of a finite difference scheme for a singularly perturbed problem with two small parameters, Report MATH-NU-18, TU Dresden, 2002.

[6] T. Linß, Layer adapted meshes for convection-diffusion problems, Comput. Meth. Appl. Mech. Eng., 192 (2003), pp. 1061-1105.

[7] N. Madden and M. Stynes, A uniformly convergent method for a coupled system of two singularly perturbed linear reaction-diffusion problems, Preprint No. 1, School of Math., University of Cork, 2002. 
[8] R. E. O'Malley, Two-parameter singular perturbation problems for second-order equations, J. Math. Mech., 16 (1967), pp. 1143-1164.

[9] E. O' Riordan, M.L. Pickett, and G.I. Shishkin, Singularly perturbed problems modelling reactionconvection-diffusion processes, Report MS-02-13, Dublin City University, 2002.

[10] H.-G. Roos and H. Zarin, The streamline-diffusion method for a convection-diffusion problem with a point source, J. Comp. Appl. Math., 150 (2003), pp. 109-128.

[11] H.-G. Roos and H. Zarin, A second order scheme for singularly perturbed differential equations with discontinuous source term, East-West J. Numer. Math., 10 (2002), pp. 275-289.

[12] G. I. Shishkin and V. A. Titov, A difference scheme for a differential equation with two small parameters at the derivatives, Numer. Meth. Contin. Medium Mech., 7 (1976), No. 2, pp. 145-155.

[13] M. Stynes and L. Tobiska, A finite difference analysis of a streamline diffusion method on a Shishkin mesh, Numer. Algorithms, 18 (1998), pp. 337-360.

[14] R. Vulanovic, A higher-order scheme for quasilinear boundary-value problems with two small parameters, Computing, 67 (2001), pp. 287-303.

Received 26 Nov. 2002

Revised 02 Jun. 2003 